\title{
Praktische Methoden der quantitativen Beschreibung des Elektrenkephalogramms.
}

\author{
Von \\ Kōiti Motokawa. \\ (本川弘 $\rightarrow$ ) \\ (Aus dem II. Institut für Physiologie der Kaiserlichen \\ Tohoku Universität, Sendai. \\ Vorstand: Prof.Dr. K. Motokawa.)
}

\section{Einleitung.}

Die gehirnelektrischen Erscheinungen sind ein empfindlicher und leicht zugänglicher Indikator für den funktionellen Zustand des Gehirns, und somit ist es nicht verwunderlich, dass die Elektrenkephalographie eine unentbehrliche Methode für physiologische sowie klinische Untersuchungen des Zentralnervensystems geworden ist. Abẹr wir finden sie in der medizinischen Welt noch nicht so eingebürgert wie die Elektrokardiographie, was hauptsächlich darauf zurückzuführen ist, dass das Elektrenkephalogramm (EEG) im allgemeinen viel komplizierter und somit viel schwieriger in seiner Deutung ist, als das Elektrokardiogramm. Beim letzteren ist der Sachverhalt verhältnismässig einfach, denn die einzelnen Zacken treten im normalenZustand der Versuchsperson in einer so regelmässigen Reihenfolge und mit einer jeweilig so konstanten Grösse auf, dass man einer etwaigen Veränderung im Intervall bzw. in der Grösse der einzelnen Zacken ohne Schwierigkeit eine gewisse Bedeutung zuschreiben darf.

Im Gegensatz dazu verhalten sich die Wellen des EEG besonders in ihrer Amplitude so regellos, dass das Verhalten der einzelnen Wellen für diagnostische $Z$ wecke kaum verwertbar ist. Vom statistischen Standpunkt aus betrachtet, richten sich die Wellen aber nach einer bestimmten Gesetzmässigkeit, wie von Motok a wa und $\mathrm{Mita}^{1{ }^{2)}}$ nachgewiesen worden ist, so dass sich das EEG nur mit geeigneten statistischen Grössen richtig kennzeichnen lässt, unter denen in erster Linie 
die durchschnittliche Periode oder Frequenz und die mittlere Amplitude zu nennen sind. Da die erstere im allgemeinen leicht zu ermitteln ist, hat sie von jeher in der Praxis mehrfach Verwendung gefunden, um damit das EEG zu charakterisieren, aber die mittlere Amplitude ist trotz ihrer Wichtigkeit in der Literatur viel seltener zu finden, was vielleicht darauf zurückzufuhren ist, dass die Ermittelung dieser Grösse viel mühsamer ist, als die der durchschnittlichen Periode oder Frequenz, insbesondere bei einer schwachen Verstärkung des Potentials.

Aus diesem Grunde versucht man das EEG mit der Schwankungsbreite der Amplituden zu kennzeichnen; diese Grösse stellt jedoch keinen zuverlässigen Massstab für die Amplitude dar, denn die letztere kann nach dem Wahrscheinlichkeitsprinzip, dessen Gültigkeit für die gehirnelektrischen Erscheinungen schon von Motokawa und Mita nachgewiesen wurde, wenigstens theoretisch alle Werte von $0 \sim \infty$ einnehmen.

Andererseits ist von amerikanischen Autoren wie H. Davis, P. A. Davis. Jasper, Rubin ${ }^{3)}$ und anderen eine praktische Grösse, die als "per cent time alpha" bezeichnet wird, in die Praxis eingeführt worden. Sie ist in der Tat sehr leicht zu ermitteln und sicher praktisch anwendbar, wenn auch mit einiger Beschränkung, aber sie lässt in ihrer Allgemeingültigkeit noch viel zu wünschen übrig, wie unten auseinandergesetzt werden wird. Muss man nach einer einwandfreien praktischen Grösse suchen, oder kann man uiberhaupt eine solche auffinden? Dies ist aber sehr zu bezweifeln, denn das arithmetische Mittel ist die einfachste statistische Grösse, nur kann es beim EEG wegen der Mühsamkeit seiner Ermittelung dem praktischen Zweck nicht entsprechen.

In Anbetracht dieser Verhältnisse verzichtete ich auf das Suchen nach einer anderen Grösse, sondern versuchte, eine einfachere Methode für die Ermittelung des arithmetischen Mittels der Amplituden zu flnden, um diese Grösse praktisch anwendbar zu machen. Andererseits habe ich in vorliegender Arbeit einen neuen Massstab vorgeschlagen, um damit die Stetigkeit der Erscheinungsweise der $\alpha$-Wellen kennzeichnen zu können.

\section{Methodik und Ergebnisse.}

1. Eigenschaften des Prozentzeit-Alphas.

Unter dem Prozentzeit-Alpha versteht man die Zeitdauer, während der die Wellen mit einer grösseren Amplitude als ein bestimmter Wert oder eine Schwelle auftreten, in Prozenten der gesamten Dauer, wobei 
die Schwellegeeignet gewählt werden muss. H. Davis u. P.A. D a v is betrachteten das Aufeinanderfolgen von wenigstens $3 \alpha$-Wellen mit Amplituden von über $7 \mu \mathrm{V}$ als überschwellig, aber die Wahl dieser Schwelle geschah ganz nach Willkür ohne theoretische Begründung. Durch diese Beliebigkeit in der Wahl der Schwelle wird die Allgemeingültigkeit des Prozentzeit-Alphas erheblich beeinträchtigt.

Um die Abhängigkeit des Prozentzeit-Alphas von der Schwelle aufzuklären, wurden seine Werte für eine Reihe der Schwellenwerte aus zwei EEG mit voneinander verschieden stark entwickelten $\alpha$-Wellen ermittelt, wobei je 500 Wellen eines EEG der Messung unterzogen wurden. Das Verfahren zeigt Abb. 1. Aneinanderliegende Tale der $\boldsymbol{a}$-Wellen wurden durch je eine Gerade miteinander verbunden, zu der je eine Parallele in einem bestimmten Abstand gezogen wurde, der der jeweiligen.Schwelle gleich war; dieses Verfahren liess sich in Wirklichkeit mittels eines Zirkels sehr leicht auf einmal ausführen, indem der Abstand der beiden Spitzen des Zirkels der jeweiligen Schwelle gleich gewählt wurde. Dann liess sich das Prozentzeit-Alpha als die Anzahl der überschwelligen Wellen in Prozenten der gesamten Zahl der Wellen ohne weiteres ermitteln.

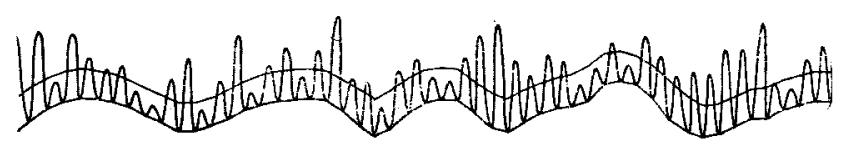

Abb. 1. Sehema für Ermittelung des Prozentzeit-Alphas.

Der Abstand zwischen den beiden Parallelen ist der Schwelle gleichzusetzen. Prozentzeit-Alpha $p=100 \times \frac{\text { Zahl der überschwelligen Wellen }}{\text { Zahl der gesamten Wellen }}$.

Das Ergebnis ist in Abb. 2 graphisch dargestellt, wobei sich die Kurve A auf das EEG mit besser entwickelten Wellen, B auf dasjenige mit schwächeren Wellen und $C$ auf das Verhältnis zwischen A und B bezieht. Wie daraus ohne weiteres hervorgeht, nimmt das ProzentzeitAlpha mit ansteigender Schwelle anfangs langsam, dann steiler und .darauf wieder langsamer ab. Das Verhältnis von A zu B ist ebenfalls von der Schwelle stark abhängig und bei einem bestimmten Wert der Schwelle, hier etwa $12 \mathrm{dem}$ Verhältnis der beiden durchschnittlichen Amplituden gleich, was darauf hindeutet, dass das Prozentzeit-Alpha bei einer geeigneten Wahl der Schwelle an Stelle der durchschnittlichen Amplitude benutzt werden kann. Handelt es sich um den Vergleich von mehreren EEG mit voneinander verschieden stark entwickelten Wellen, so kann es nicht mehr gelten, weil die Bestimmung einer 




Abb. 2. Abhängigkeit des Prozentzeit-Alphas von der Schwelle.

A : Bei einem EEG mit einer durchschnittlichen Amplitude von 18,46 mm.

B : Bei einem EEG mit einer durchschnittlichen Amplitude von 12,01 mm.

C: Verhältnis der Werte des Prozentzeit-Alphas von $A$ und $B_{a}$

Es ist an dem mit einem Kreuz markierten Punkt der Kurve dem Verhältnis der beiden durchschnittlichen Amplituden gleich.

für alle EEG zugleich geeigneten Schwelle prinzipiell unmöglich ist.

Um dieBeziehung zwischen dem Prozentzeit-Alpha und der durchschnittlichen Amplitude kennenzulernen, wurden etwa 50 EEG mit verschiedenen durchschnittlichen Amplituden auf ihr Prozentzeit-Alpha hin untersucht, wobei alle Messungen mit derselben Schwelle von 10 . $\mathrm{mm}$ ausgeführt wurden. Das Ergebnis ist in Abb. 3 graphisch dargestellt. Wie daraus ohne weiteres ersichtlich, verläuft die Kurve im grossen und ganzen S-förmig, wie etwa die Kennlinie einer Elektronenröhre, so dass die durchschnittliche Amplitude und das ProzentzeitAlpha am mittleren Stück der Kurve annähernd linear zueinander stehen. Die Lage und Steilheit der Kurve verändern sich mit der Sch-welle in der Weise, dass die Kurve, je kleiner die Schwelle gewählt wird, desto steiler ansteigt, aber die S-förmige Eigenschaft dadurch keinewesentliche Veränderung erfährt. Dies Verhältnis deutet darauf hin, dass eine innige Beziehung zwischen beiden Grössen bestehen muss, und in diesem Punkt schien der Schlüssel zur Lösung der eingangs aufgeworfenen Frage zu liegen, was mich veranlasste, diese Beziehungtheoretisch weiter zu untersuchen. 


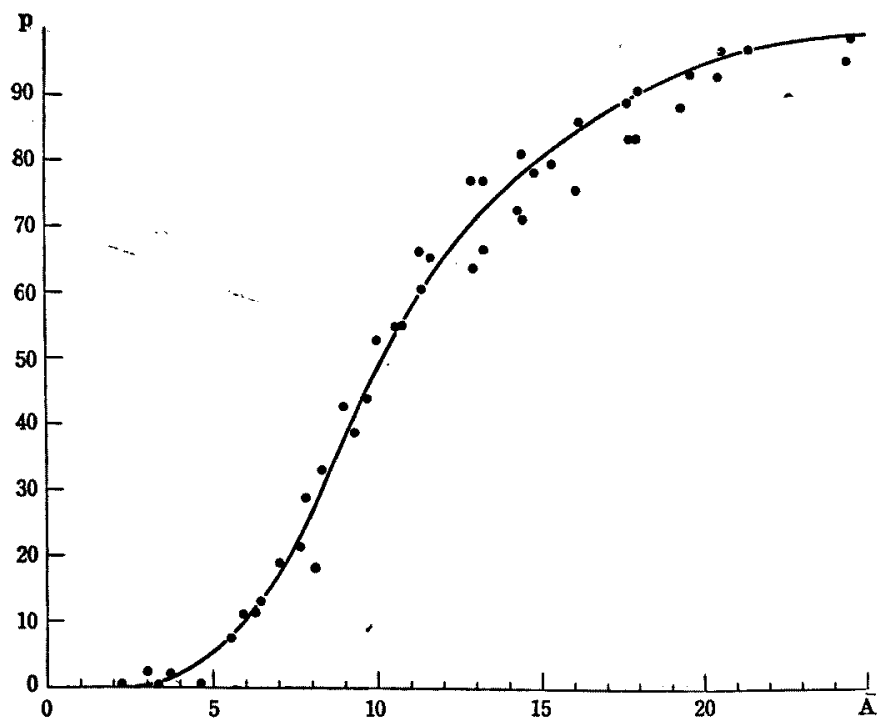

Abb. 3. Beziehung zwischen dem Prozentzeit-Alpha $p$ und der durchschnittlichen Amplitude $\bar{A}$, wobei" eine Schwelle von $10 \mathrm{~mm}$ für Ermittelung des ersteren benutzt warde. Die Eigenschaft der Kurve ändert sich nicht, wie gross die Schwelle auch gewählt werden mag.

2. Theoretische Beziehung zwischen dem ProzentzeitAlpha und der durchschnittlichen Amplitude.

Wie in unseren vorausgegangenen Arbeiten ${ }^{1) 4}$ wiederholt experimentell nachgewiesen, richtet sich die Amplitude der $\alpha$-Wellen nach dem statistischen Gesetz, nämlich :

$$
d n=2 h^{2} A N e^{-h^{2} A^{2}} d A,
$$

wobei $d n$ die Häufigkeit der Amplituden, die zwischen $A$ und $A+d \boldsymbol{A}$ liegen, $N$ die gesamte Anzahl der Wellen und $h$ eine Konstante bedeutet. Die Häufigkeit $n$ der Amplituden, die grösser als ein bestimmter Wert $s$ sind, lässt sich durch Integration der obigen Formel von $s$ bis zu $\infty$ ohne weiteres ermitteln:

$$
n=2 h^{2} \cdot N \int_{s}^{\infty} A e^{-h^{2} A^{2}} d A .
$$

Diese Integration kann man, wenn $h^{2} A^{2}=z$, also $2 h^{2} A d A=d z$ gesetzt wird, leicht ausführen in folgender Weise, nämlich :

$$
n=N \int_{h^{2} s^{2}}^{\infty} e^{-z} d z=N\left[-e^{-z}\right]_{h^{2} s^{2}}^{\infty}=N e^{-h^{2} s^{2}}
$$


Die Zeit, während deren die Amplitude überschwellig bleibt, muss also gleich $N \tau e^{-h^{2} s^{2}}$ sein, vorausgesetzt, dass die Periode der $\alpha$-Wellen $\tau$ Sek. beträgt, und das Prozentzeit-Alpha ergibt sich daraus ohne weiteres folgendermassen:

$$
p=100 \frac{N \tau e^{-h^{2} s^{2}}}{N \tau}=100 e^{-h^{2} s^{2}},
$$

Andererseits besteht zwischen dem arithmetischen Mittel $\bar{A}$ der Amplituden und der Konstante $h$ die Beziehung:

$$
h=\frac{\sqrt{\pi}}{2 \bar{A}},
$$

wie in unseren früheren Arbeiten ${ }^{1) 4}$ nachgewiesen wurde. Aus (2) und (3) ergibt sich ohne weiteres die Beziehung:

$$
p=100 e^{-\frac{\pi}{4 \bar{A}^{2}} s^{2}}
$$

oder

$$
\bar{A}=\sqrt{\frac{0,3412 s^{2}}{2-\log p}}
$$

und aus dieser Beziehung lässt sich die durchschnittliche Amplitude aus dem leichter ermittelbaren Prozentzeit-Alpha berechnen. Die so berechneten Werte sind in Tabelle I wiedergegeben. Wie daraus ohne weiteres hervorgeht, sind die Werte des Prozentzeit-Alphas, aus denen die durchschnittliche Amplitude berechnet wurde, je nach Wahl der

$$
\text { Tabelle I. }
$$

Die aus dem Prozentzeit-Alpha theoretisch berechneten Werte der durchschnittlichen Amplitude. Der aus wirklicher Messung erhaltene Wert betrug $18,46 \mathrm{~mm}$.

\begin{tabular}{c|c|c|c}
\hline $\begin{array}{c}\text { Schwelle } \\
\text { in mm }\end{array}$ & $\begin{array}{c}\text { Prozentzeit- } \\
\text { Alpha }\end{array}$ & $\begin{array}{c}\text { Berechnete } \\
\text { Werte in mm }\end{array}$ & $\begin{array}{c}\text { Abweichung } \\
\text { von 18,46 }\end{array}$ \\
\hline 2 & 98,6 & 15 & $-3,46$ \\
7 & 92,2 & 21,5 & $+3,04$ \\
12 & 77 & $20 ; 8$ & $+2,34$ \\
17 & 52,8 & 18,9 & $+0,44$ \\
21 & 38,2 & 19,0 & $+0,54$ \\
22 & 36,6 & 19,4 & $+0,94$ \\
27 & 14 & 17,1 & $-1,36$ \\
32 & 3,8 & 15,7 & $-2,76$ \\
37 & 1,2 & 15,2 & $-3,26$
\end{tabular}


Schwelle voneinander stark verschieden, die berechneten Werte stehen dagegen einander sehr nahe. Besonders bemerkenswert ist, dass sie dann mit dem wirklich gefundenen Wert befriedigend übereinstimmen, wenn dieSchwelle in der Weise gewählt wird, dass sie der durchschnittlichen Amplitude nahe kommt, oder was dasselbe ist, dass das Prozentzeit-Alpha zwischen etwa 30-60 fällt.

\section{Praktische Methode für Ermittelung der durchschnittlichen Amplitude.}

Die obige Rechnung kann aber für Praktiker etwas lästig und auch zaitraubend sein, so dass ich versucht habe, sie in folgender Weise entbehrlich zu machen. Es wurden nach Gleichung (4) $s$-p-Diagramme für verschiedene Werte der durchschnittlichen Amplitude konstruiert, damit man aus gegebenen Werten von $s$ und $p$ auf graphischem Wege die entsprechende durchschnittliche Amplitude ermitteln kann, wie in Abb. 4 dargestellt ist.

Die praktische Methode lässt sich also in folgender Weise zusammenfassen:

(i) Die Schwelle für die Messung des Prozentzeit-Alphas muss nach dem Augenmass möglichst nahe dem arithmetischen Mittel der Amplituden gewählt werden.

(ii) Die Zahl der überschwelligen Wellen muss in Prozenten der gesamten Anzahl der Wellen, die wenigstens 100 betragen muss, ausgedrückt werden. Falls das so ermittelte Prozentzeit-Alpha zu niedrig ist, wie z. B. unter etwa 30 oder zu hoch wie z. B. über etwa 60 , so muss man mit einer kleineren resp. grösseren Schwelle wiederum das Prozentzeit-Alpha ermitteln, damit es zwischen 30-60 fällt. Aber die Wahl der Schwelle gelingt im allgemeinen so leicht, dass diese Massnahme in Wirklichkeit kaum in Frage kommt.

(iii) Man muss nun auf den $s$ - $p$-Diagrammen in Abb. 4 den der so gewählten Schwelle $s$ und dem so ermittelten Prozentzeit-Alpha $p$ entsprechenden Punkt auffinden und sehen, zu welcher Kurve er gehört. Wenn er z. B. zwischen den beiden Kurven 28 und 29 liegt, so muss die durchschnittliche Amplitude zwischen 28 und 29 liegen, wobei die Einheit des abgelesenen Wertes derjenigen der Schwelle gleich ist. Der Bruchteil lässt sich durch die gewöhnliche Interpolationsmethode leicht bestimmen; wenn z. B. der Punkt von den beiden Kurven im Verhältnis $3: 7$ entfernt liegt, so ist der Wert ohne weiteres als 28,3 abzulesen.

Falls die Schwelle kleiner als 4 gewählt werden muss, wofür kein $s$-p-Diagramm in Abb. 4 gegeben ist, so muss man den Schwellenwert 
mit 10 multiplizieren und den dementsprechenden -Wert der durchschnittlichen Amplitude ermitteln. Dann ist der aufzufindende Wert einem Zehntel des so erhaltenen gleich.

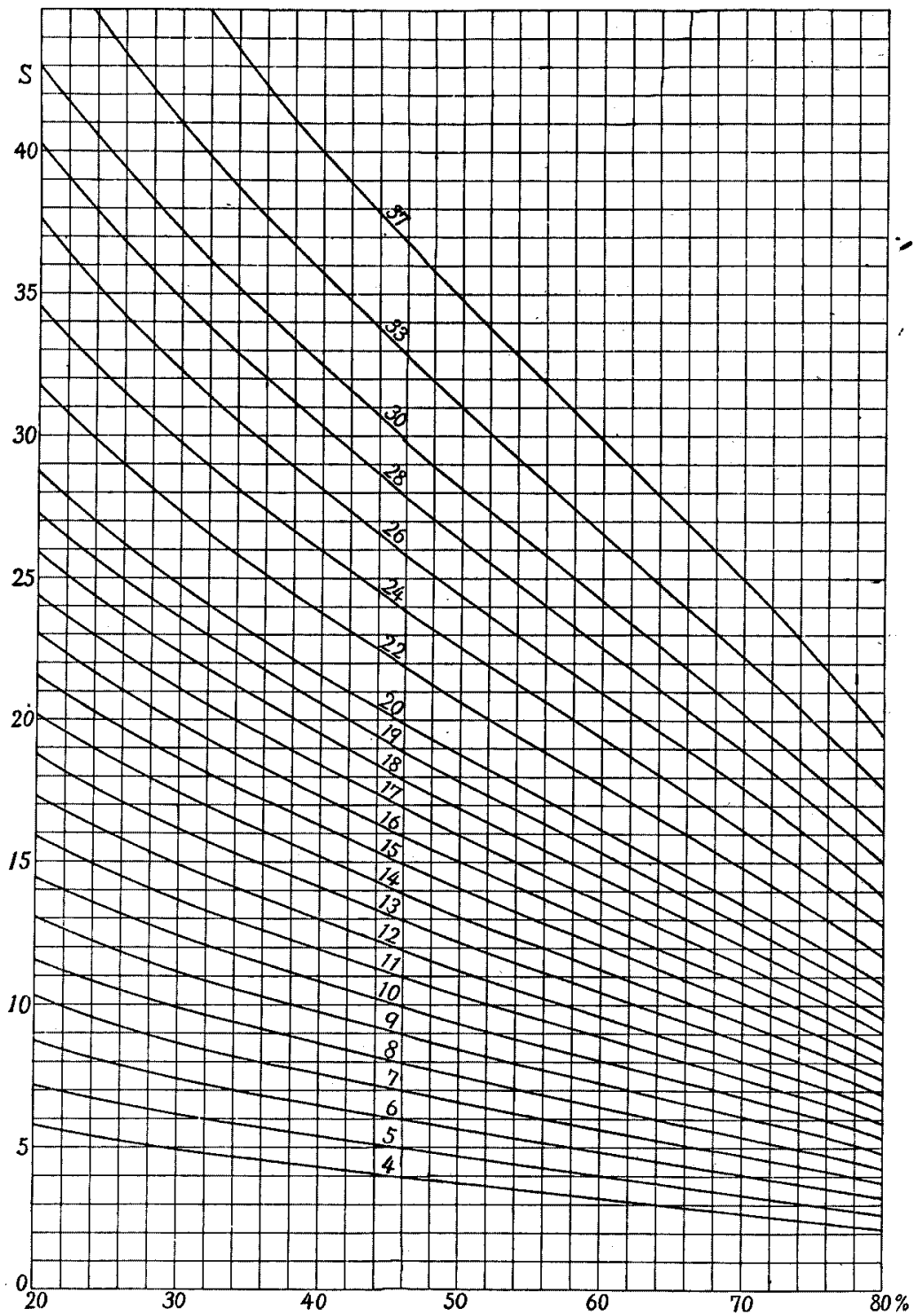

Abb. 4. s-p-Diagramme für Ermittelung der durchschnittlichen

Amplitude aus dem Prozentzeit-Alpha. Erklärung im Text. 
Diese Methode hat sich in der Tat nicht nur für die normalen sondern auch für die pathologischen EEG ausgezeichnet bewährt. In Tabelle II sind die nach dieser Methode erhaltenen Werte $\overline{A p}$ und die wirklich ermittelten $\bar{A}$ der durchschnittlichen Amplitude nebeneinander dargestellt. Wie daraus ohne weiteres hervorgeht, bleibt die Differenz zwischen beiden in den meisten Fällen innerhalb des Messungsfehlers, nämlich innerhalb einiger Bruchteile von $1 \mathrm{~mm}$.

$$
\text { Tabelle } \Pi \text {. }
$$

Übereinstimmung zwischen den nach der praktischen Methode ermittelten Werten $\overrightarrow{A p}$ und den aus wirklicher Messung erhaltenen $\vec{A}$ der durchschnittliehen Amplitude.

\begin{tabular}{|c|c|c|c|c|c|c|}
\hline \multicolumn{3}{|c|}{ Bei normalen EEG } & \multicolumn{4}{|c|}{ Bei pathologischen EEG } \\
\hline $\begin{array}{c}\text { Versuchs: } \\
\text { person }\end{array}$ & $\begin{array}{c}\bar{A} p \\
\text { in } \mathrm{mm}\end{array}$ & $\begin{array}{c}\bar{A} \\
\text { in } \mathrm{mm}\end{array}$ & $\begin{array}{c}\text { Versuchs- } \\
\text { person }\end{array}$ & $\begin{array}{c}\bar{A} p \\
\text { in } \mathbf{m m}\end{array}$ & in $\overline{\mathrm{mm}}$ & Krankheit \\
\hline Miyake & 18,3 & 18,66 & Nanba & 8,2 & 7,78 & Traum. Epilep. \\
\hline Minra & 17,1 & 16,57 & Saito & 16,0 & 16,49 & Genuin. Epilep. \\
\hline Minegisi & 10,7 & 11,44 & Honda & 25,5 & 25,35 & Postencephal. Epilep. \\
\hline Narita & 7,6 & 7,24 & Kasuya & 18,3 & 19,91 & Jackson: Epil. \\
\hline Hongo & 13,3 & 12,94 & Oka & 10,5 & 11,28 & Genuin. Epil. \\
\hline Endo & 10,0 & 9,79 & Nisizawa & 10,3 & 10,17 & Jackson. Epil. \\
\hline Sasaki & 9,6 & 9,02 & Onodera & 14,1 & 13,46 & Genuir. Epil. \\
\hline Tsuzignti & 12,7 & 13,10 & Kisinami & 31,5 & 31,94 & Jackson. Epil. \\
\hline
\end{tabular}

Es handelt sich bei dieser Methode nur um die Entscheidung, ob die einzelnen Amplituden überschwellig sind oder nicht, aber nicht um ihre absolute Grösse, so dass man auch bei EEG mit nur schwach entwickelten $\alpha$-Wellen, bei denen die Messung der einzelnen Amplituden sehr erschwert ist, verhältnismässig leicht die durchschnittliche Amplitude ermitteln kann, was den sonst unmöglichen quantitativen Ausdruck des EEG ohne weiteres ermöglicht. Der wesentliche Vorteil dieser Methode besteht allerdings darin, dass sie uns vor allem so viel Mühe und Zeit spart, dass wir sie ohne weiteres praktisch anwenden können.

\section{Kontinuität des EEG.}

Die Erscheinungsweise der $\alpha$-Wellen lässt sich allein mit der durchschnittlichen Amplitude und der durchschnittlichen Periode nicht vollkommen kennzeichnen, was aus den Schemata in Abb. 5 ohne weiteres verständlich ist, denn die dort gezeichneten dreierlei Kurven besitzen 
alle dieselbe durchschnittliche Amplitude undFrequenz, sind aber in der Erscheinungsweise der Wellen doch voneinander stark verschieden, Dieser Unterschied bezieht sich auf die Reihenfolge der verschiedenen Amplituden, oder allgemeiner ausgedruickt, darauf, wie lang ein bestimmter Zustand dauern kann, und nimmt einen geeigneten quantitativen Ausdruck in Anspruch.

(i)

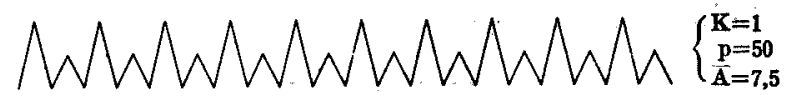

(ii)

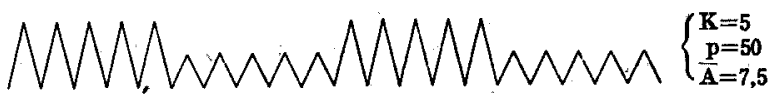

(iii)

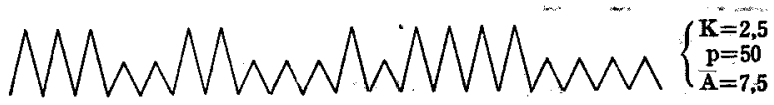

Abb. 5. Bedeutung der ,Kontinuität" (schematische Darstellung). Die hier dargestellten drei Kurven, denen dasselbe Prozentzeit-Alpha und dieselbe durchschnittliche Amplitude zukommen, unterscheiden sich voneinander nur durch die Verschiedenheit ihrer Kontinuität.

In der Physik ${ }^{5 /}$ macht man als quantitativen Ausdruck dieser Eigenschaft von einer als ,durchschnittliche Dauer“ bezeichneten Grösse Gebrauch, die aus einer Reihe der Messungswerte in folgender Weise ermittelt wird. Ist die Reihe, bei welcher das Intervall der Messung $\tau$ Sek. beträgt, z. B.

$$
\mathrm{Nx}, \underbrace{\mathrm{x}, \mathrm{x}, \mathrm{x}}, \mathrm{Nx}, \mathrm{Nx}, \underbrace{\mathrm{x}, \mathrm{x}}, \mathrm{Nx}, \underbrace{\mathrm{x}, \mathrm{x}, \mathrm{x}}, \mathrm{Nx}, \underbrace{\mathrm{x}}, \mathrm{Nx}, \cdots \cdots \cdots \cdots \cdot
$$

wobei $x$ den betreffenden Zustand und $N x$ alle anderen Zustände (Nicht $\mathrm{x})$ bedeutet, so wird die gesamte Anzahl von $\mathrm{x}$ in erster Linie aufgefunden, mit dem Intervall $\tau$ multipliziert und dann durch die Anzahl aller Gruppen'von x dividiert. Betrachtet man z. B. die grösseren Wellen in $\mathrm{Abb} .5$ als den $\mathrm{x}-\mathrm{Z}$ ustand und die kleineren als $\mathrm{Nx}$, so beträgt die durchschnittliche Dauer für $\mathrm{x}$ bei der Kurve (i) nur $\tau$, bei (ii) $5 \tau$ und bei (iii) $2,5 \tau$ Sek.

Was die $\alpha$-Wellen des EEG anbetrifft, so bleibt die Amplitude anders als bei diesen Schemata für keinen Augenblick konstant, sondern schwankt regellos in weitem Umfang. Aus diesem Grund ist die durchschnittliche Dauer für eine bestimmte Grösse der Amplitude im allgemeinen sehr kurz und zeigt keinen auffallenden Unterschied je nach EEG an, so dass man mit dieser Grösse selbst einzelne EEG kaum zu kennzeichnen vermag. Denkt man sich aber unter den $x-$ Zustand verschiedene Amplituden über eine gewisse Grenze mitein- 
begriffen, so wird die durchschnittliche Dauer länger, und somit kommt der Unterschied je nach EEG dementsprechend deutlicher zum Ausdruck ; man braucht dabei nur eine geeignete Schwelle zu wählen, um die überschwelligen Amplituden als $\mathrm{x}$ und die unterschwelligen als $\mathrm{Nx}$ betrachten zu können. Die Schwelle muss eine geeignete und für alle Fälle gemeinsame Grösse sein, denn die durchschnittliche Dauer muss desto länger ausfallen, je kleiner die Schwelle gewählt wird, aber es handelt sich hierbei nicht um die absolute sondern um die zur durchschnittlichen Amplitude relative Grösse der Schwelle.

Ich möchte aus diesem Grunde die durchschnittliche Amplitude selbst, welche sich nach der oben erläuterten Methode immer leicht ermitteln lässt, als. Schwelle wählen. Obgleich die durchschnittliche Dauer ihrer Definition nach das Intervall oder die Periode enthält, so ist doch letztere wenigstens beim EEG überflüssig, weil man bei der Beschreibung des EEG die Periode oder Frequenz gesondert angeben muss. Ich möchte also die Periode aus der durchschnittlichen Dauer fortfallen lassen und die so entstandene Grösse als „Kontinuität" bezeichnen. Diese ist also der Anzahl der. Amplituden, die grösser als die durchschnittliche Amplitude sind, dividiert durch die Anzahl ihrer Gruppen gleich und zeigt an, wieviel übérschwellige Wellen im Durchschnitt aufeinanderfolgen.

In Tabelle III sind Beispiele der Kontinuität wiedergegeben, die aus den EEG der verschiedenen Rindenteile einer Anzahl Versuchspersonen stammten, und darin sind auch die Werte des ProzentzeitAlphas, welche mit der durchschnittlichen Amplitude als Schwelle ermittelt wurden, nebeneinander dargestellt. Unter dieser Bedingung,

Tabelle III.

Kontinuität $\boldsymbol{K}$ und Prozentzeit-Alpha $p$ der EEG aus versehiodenen Rindenregionen von 6 Versuchspersonen. Die Schwelle für Messung des Prozentzeit-Alphas wurde der durchschnittlichen Amplitude gleichgesetzt.

\begin{tabular}{|c|c|c|c|c|c|c|c|c|c|c|c|c|}
\hline \multirow{2}{*}{$\begin{array}{l}\text { Rinden- } \\
\text { region }\end{array}$} & \multicolumn{2}{|c|}{ Narita } & \multicolumn{2}{|c|}{ Minegisi } & \multicolumn{2}{|c|}{ Endo } & \multicolumn{2}{|c|}{ Sasaki } & \multicolumn{2}{|c|}{ Miura } & \multicolumn{2}{|c|}{ Tuziguti } \\
\hline & $p$ & $K$ & $p$ & $K$ & $p$ & $K$ & $p$ & $K$ & $p$ & $K$ & $p$ & $K$ \\
\hline Occipit. & 47 & 3,62 & 45 & 1,8 & 46 & 1,77 & 48 & 1,92 & 45 & 1,73 & 43 & 2,15 \\
\hline Pariet. & 45 & 2,04 & 45,5 & 1,82 & 45 & 1,39 & 48 & 2,66 & 47 & 1,81 & 44 & 1,76 \\
\hline Praecent. & 48 & 2,28 & 45 & 1,73 & 48 & 2,52 & 44 & 1,83 & 46 & 1,96 & 45 & 1,96 \\
\hline Frontal. & 47 & 2,47 & 48 & 2,18 & 45 & 2,04 & 47 & 1,96 & 45 & 2,36 & 51 & 2,68 \\
\hline Mittel & 46,8 & 2,60 & 45,9 & 1,88 & 45,9 & 1,93 & 46,8 & 2,09 & 45,8 & 1,97 & 45,8 & 2,14 \\
\hline
\end{tabular}


d.h. $s=\bar{A}$, muss das Prozentzeit-Alpha immer einen bestimmten Wert annehmen, denn $p=100 e^{-\frac{\pi}{4} \frac{s^{2}}{\bar{A}^{2}}}=100 e^{-\frac{\pi}{4}}=45,6$, und in der Tat stimmen die in der Tabelle angegebenen Werte in allen Fällen mit dem theoretischen Wert befriedigend überein. Im Gegensatz dazu sind die Werte der Kontinuität von Individuum zu Individuum und auch selbst bei derselben Versuchsperson von EEG zu EEG voneinander ziemlich stark verschieden. Diese Verhältnisse deuten darauf hin, dass dieKontinuität mehrfach vom jeweiligen psychischen Zustand der Versuchsperson abhängt. Es ist besonders hervorzuheben, dass trotz dieser $\mathrm{Ab}$ hängigkeit vom jeweiligen Zustand ziemlich deutliche individuelle Unterschiede voṛhanden sind, die man als Ausdruck der individuellen Eigentümlichkeiten der psychischen Zustände betrachten dürfte. Die EEG von Narita, die immer besonders hohe Werte der Kontinuität aufwiesen, liessen sich ohne besondere Messung von anderen unterscheiden. Welche Bedeutung der Kontinuität zukommt, muss aber künftigen Untersuchungen überlassen werden.

\section{Schlussbetrachtung.}

Die Feststellung der Beschreibungsmethode stellt die wichtigste Voraussetzung zur Förderung des in Frage stehenden Gebietes der Wissenschaft dar. Wenn sie einmal festgesetzt worden ist, ist es sehr wünschenswert, dass alle Forscher übereinstimmend dieselbe Beschreibungsmethode benutzen. In den bisherigen Arbeiten über die gehirnelektrischen Erscheinungen pflegte man einige Stücke des EEG zu illustrieren und hierzu eine nur qualitative Beschreibung hinzusetzen. Man mag die Illustration der Bilder an sich für dìe überzeugendste Methode halten und andere sprachliche oder zahlenmässige Beschreibungsmethoden nur als Hilfsmittel oder nur der Raumersparnis halber als wichtig betrachten, was aber wenigstens beim EEG nicht zutrifft. Es ist nur ein Rohmaterial wie ein Erz und lässt sich somit ohne geeignete Analyse nicht verwerten. Darüber hinaus kann die Illustration von nicht richtig gewählten Stücken eines EEG sogar irreführen, denn die Erscheinungsweise der Wellen ist selbet bei ein und derselben Versuchsperson oder -tier unter denselben Versuchsbedingungen von Zeit zu Zeit im allgemeinen so stark verschieden, dass man, wenn man will, mehrere Stücke verschiedener Bilder aus ein und demselben EEG beliebig wiedergeben kann. Hätte man unter diesen nur dasjenige, dessen Bild der eigenen Meinung entspricht, ausgewählt und abgebildet, so müsste es sehr irreführend sein. 
Es hat sich herausgestellt, dass sich das EEG mit den oben genannten dreierlei Grössen, nämlich der durchschnittlichen Amplitude, der Kontinuität und der durchschnittlichen Frequenz praktisch kennzeichnen lässt. Bei der Beschreibung mit diesen statistischen Grössen kann man im Gegensatz zu der Illustration durch Bilder allein nicht nur viel Raum sparen, sondern der oben erläuterten Gefahr erfolgreich vorbeugen.

Es können ausser den genannten drei Hauptmerkmalen unter Umständen noch andere wie z. B. die Wellenform, die Schwankungsweise der Grundlinie, das Mischungsverhältnis verschiedener Arten der Wellen usw. in Frage kommen, und auch für sie wird die Festsetzung der Beschreibungsmethode in Anspruch genommen werden. Aber ich möchte sie den künftigen Untersuchungen der einzelnen Fälle, wo solche Merkmale in Frage kommen werden, überlassen und hoffen, dass wenigstens die drei Hauptmerkmale als die wichtigsten quantitativen
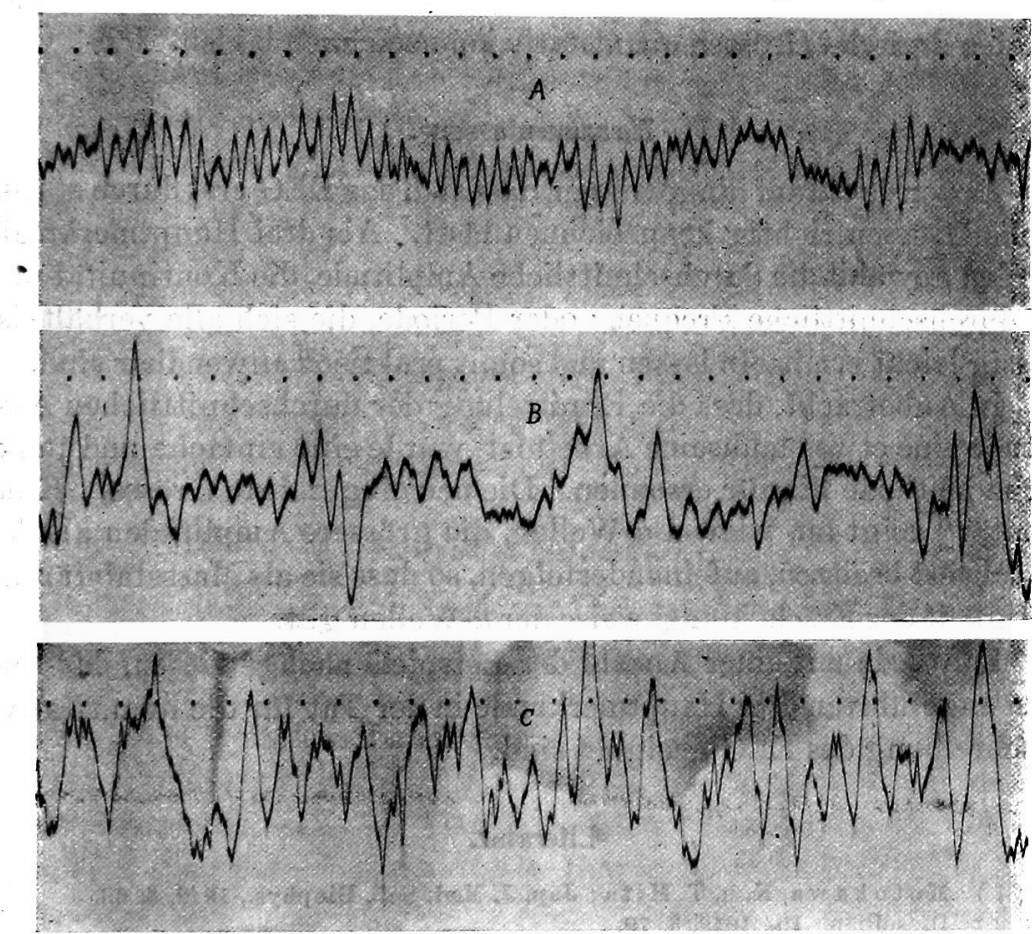

Abb. 6. EEG mit verschiedenen Hauptmerkmalen.

A : Bei psychischer Passi vität eines normalen Menschen.

B : In leicht erregtem Zustand eines anderen normalen Menschen.

C: Im psychischen Ruhezustand eines Epileptikers. 
Tabelle IV.

Die Hauptmerkmale der EEG in Abb. 6 .

\begin{tabular}{c|c|c|c|c|c}
\hline \multirow{2}{*}{$\begin{array}{c}\text { Versuchs- } \\
\text { person }\end{array}$} & $\begin{array}{c}\text { Durchschnitt. } \\
\text { Amplitude in } \mu \mathrm{V}\end{array}$ & \multicolumn{2}{|c|}{ Durchsehnitt. Frequenz } & Kontinuität & Bemerkung \\
\cline { 3 - 5 } & & $\alpha$ od. $\delta$ & $\beta$ & & \\
\hline (A) Narita & 17,39 & 10,61 & 21,4 & 3,92 & Normal \\
(B) Hongo & 18,52 & 7,88 & 22,0 & 1,62 & Normal \\
(C) Honda & 44,35 & 4,35 & 28,5 & 1,56 & Postence. \\
& & & & & phal. Epil.
\end{tabular}

Massstäbe für das EEG im ganzen Kreis der elektrenkephalographischen Forschung Anwendung finden wird.

$\mathrm{Zu}$ guter Letzt seien einige Beispiele der EEG und der dementsprechenden Hauptmerkmale in Abb. 6 und Tabelle IV wiedergegeben. Man wird sich aus dem Vergleich der Abb. mit den Daten in der Tabelle überzeugen können, wie befriedigend sich die Unterschiede der Bilder mit den dreierlei Grössen quantitativ kennzeichnen lassen.

\section{Zusammenfassung.}

Es wurde darauf hingewiesen, dass sich das EEG erst durch statistische Grössen richtig kennzeichnen lässt. Als drei Hauptmerkmale wurden gewählt die durchschnittliche Amplitude, die Kontinuität und die durchschnittliche Frequenz oder Periode, die sich alle verhältnismässig leicht ermitteln lassen und somit praktisch anwendbar sind.

In Anbetracht, dass die Ermittelung der durchschnittlichen Amplitude eine etwas mühsame Arbeit ist, wurde eine einfache und praktische Methode hierfür ersonnen. Die neu eingeführte Grösse, „Kontinuität" zeigt an, wieviel $\alpha$-Wellen, die grössere Amplituden als der Mittelwert besitzen, aufeinanderfolgen, so dass sie als Massstab für die Stetigkeit der Erscheinungsweise der $\alpha$-Wellen gilt.

Es wurde mit einer Anzahl der Beispiele nachgewiesen, dass die oben angeführten drei Hauptmerkmale in der Tat für die quantitative Beschreibung des EEG geeignet sind.

\section{Literatur.}

( I) Motokawa, K. u. T. Mita; Jap. J. Med. Sci., Biophys., 1942, 8, 63.

(2) Dieselben; Ib., 1942, 8, 79.

(3) Ru bi n, M. A.; J. Neurophysiol., 1938, 1, 313.

(4) Motokawa, K.; Tohoku J. Exp. Med., 1943, 15, 278.

(5) F ürth, R.; Schwankungserscheinungen in der Physik (1920). 